\title{
Evaluation of a technique to measure heart rate variability in anaesthetised cats
}

\begin{abstract}
Analysis of heart rate (HR) and heart rate variability (HRV) are powerful tools to investigate cardiac diseases, but current methods, including 24-h Holter monitoring, can be cumbersome and may be compromised by movement artefact. A commercially available data capture and analysis system was used in anaesthetised healthy cats to measure HR and HRV during pharmacological manipulation of HR. Seven healthy cats were subjected to a randomised crossover study design with a 7 day washout period between two treatment groups, placebo and atenolol $(1 \mathrm{mg} / \mathrm{kg}$, IV), with the efficacy of atenolol to inhibit 61 adrenoreceptors challenged by epinephrine. Statistical significance for the epinephrine challenge was set at $\mathrm{P}$ $<0.0027$ (Holmï Bonferroni correction), whereas a level of significance of $\mathrm{P}<0.05$ was set for other variables.

Analysis of the continuous electrocardiography (ECG) recordings showed that epinephrine challenge increased HR in the placebo group $(\mathrm{P}=0.0003)$ but not in the atenolol group. The change in HR was greater in the placebo group than in the atenolol group $(\mathrm{P}=0.0004)$. Therefore, compared to cats pre-treated with placebo, pre-treatment with atenolol significantly antagonised the tachycardia while not significantly affecting HRV. The increased HR in the placebo group following epinephrine challenge was consistent with a shift of the sympathovagal balance towards a predominantly sympathetic tone. However, the small (but not significant at the critical value) decrease in the normalised high-frequency component (HFnorm) in both groups of cats suggested that epinephrine induced a parasympathetic withdrawal in addition to sympathetic enhancement (increased normalised low frequency component or LFnorm). In conclusion, this model is a highly sensitive and repeatable model to investigate HRV in anaesthetised cats that would be useful in the laboratory setting for short-term investigation of cardiovascular disease and subtle responses to pharmacological agents in this species.
\end{abstract}

Keyword: Heart rate; Heart rate variability; Autonomic nervous system; Cat; Electrocardiography 